\title{
Genetic Variability, Correlation and Path Coefficient Studied for Yield and Quality Traits in JNPT Lines of Rice (Oryza sativa L.)
}

\author{
Shivangi Rahangdale, Yogendra Singh*, G.K. Koutu and S. Tiwari \\ Department of Plant Breeding and Genetics, Jawaharlal Nehru Krishi Vishwa Vidyalaya \\ Jabalpur-482004, Madhya Pradesh, India \\ *Corresponding author
}

\begin{tabular}{|l|}
\hline Ke y w o r d s \\
Genetic variability, \\
Correlation and \\
path coefficient, \\
JNPT Lines \\
Oryza sativa
\end{tabular}

\section{A B S T R A C T}

In present study, sixty-seven JNPT (Jawahar New Plant Type) lines were evaluated for twenty-eight morphological and quality traits planted in Randomized Complete Block Design with three replications. The experiment was conducted during kharif seasons of 2018 at Seed Breeding Farm, Department of Plant Breeding and Genetics, College of Agriculture, JNKVV, Jabalpur (M.P.), India. Observations were recorded on the basis of middle five random competitive plants selected from each line in every replication for yield and quality traits to study the genetic variability parameters, correlation coefficient and path coefficient for yield and its attributing traits. The values of PCV for all the traits were found to be more than GCV and very small difference was present in between GCV and PCV revealing little influence of environment for their expression. High heritability accompanied with high genetic advance was observed for the traits thousand grain weight $(\mathrm{g})$, head rice recovery $(\%)$, spikelet fertility $(\%)$, harvest index $(\%)$, number of spikelets/panicle, fertile spikelets/panicle, spikelet density, grain yield /plant (g), panicle weight/plant (g), number of tillers/plant and number of productive tillers/plant. Based on the results from correlation and path coefficient analysis, it is concluded that number of tillers/plant, spikelet fertility and panicle weight/plant showed positive correlation with grain yield and at same time exhibited high positive direct effect towards yield. Therefore, they seem to be primary yield contributing character and thus can be used as direct selection to improve genetic yield potential of rice.

\section{Introduction}

Rice (Oryza sativa L.) is one of the most important crops that feed more than half the population of the world. The biggest consuming areas are Asia, Sub-Saharan Africa, and South America. Globally, after China, India is first in rice cultivation and second in rice production. It adds $26.9 \%$ of rice production globally. Rice occupies onequarter of the total crop area within the country, contributes around 40 to 43 per cent of total food grain production, and continues to play an important role in the domestic food and livelihood security framework. In Asia, $90 \%$ of all rice is cultivated and eaten. World 
paddy production has steadily increased from around 220 million tons in 1960 to over 759.6 million tons in 2017, and rice production in 2017 is 503.9 million tons. At this level, global production would exceed the 2016 record by a modest $0.6 \%$ or 4.5 million tons (FAOSTAT, 2018).

The present investigation was undertaken to gather some useful information on genetic variability character association and path coefficient analysis in a set of sixty-seven JNPT lines of rice genotypes. Heritability $\left(\mathrm{h}^{2}\right)$ is an index of transmissibility of the characters from the parents to offspring and has a predictive role in crop breeding programs (Khan and Naqvi, 2011). Improvement in the mean genotypic value of selected plants over the parental population is known as genetic advance. The genetic advance for the studied traits is dependent on the extent of genetic variability, heritability and selection intensity (Allard, 1960). It is not necessary that a character showing high heritability will also exhibit high genetic advance (Johnson, et al., 1955). Selection of parents based on yield alone is often misleading. Hence, knowledge about relationship between yield and its contributing characters is needed for an efficient selection strategy for the plant breeders to evolve an economic variety. Correlation studies provide better understanding about yield contributing characters. Path coefficient analysis provide information about influence of each contributing trait to yield directly as well as indirectly and also enables breeders to rank the genetic attributes according to their contribution.

\section{Materials and Methods}

The experimental material consists of 67 new JNPT lines (Table 1) derived from Indica $X$ Japonica subspecies crosses $\left(\mathrm{F}_{8}-\mathrm{F}_{9} \quad \mathrm{BC}_{3}\right.$ generation) developed by Rice Improvement Project, JNKVV, Jabalpur were grown during
Kharif season of 2018 at Seed Breeding Farm, JNKVV, Jabalpur (M.P.), India. These lines were planted in Randomized Complete Block Design with three replications. Twenty-one days old seedlings were transplanted in the experimental site with spacing of $15 \mathrm{~cm}$ between plant to plant and $20 \mathrm{~cm}$ between the rows, keeping single seedling per hill. Gap filling was done within a week so as to keep uniform plant population. Fertilizer dose of $120 \mathrm{~kg} \mathrm{~N}, 60 \mathrm{~kg} \mathrm{P}_{2} \mathrm{O}_{5}$ and $60 \mathrm{~kg} \mathrm{~K}_{2} \mathrm{O}$ was applied. The standard agronomic practices were adopted for normal crop growth.

Twenty-eight quantitative and quality characteristics were used to characterize and assess the genetic diversity of JNPT lines as per rice DUS guideline. Five plants were randomly chosen from each replication in each genotype for yield assessment and quality attribute traits. The observations were recorded as per the standard procedure and subjected to statistical analysis using mean values of five randomly selected plants.

\section{Statistical analysis}

The data on quantitative characters were statistically analysed on the basis of model described by Cochran and Cox (1950) for randomized complete block design. Parameters of genetic variability include mean, range, genotypic and phenotypic coefficient of variation were calculated by the method suggested by Burton (1952) and the estimate of PCV and GCV were classified as low, moderate and high according to Sivasubramanian and Madhavamenon (1973).

Heritability for broad sense is the ratio of genotypic variance to the total phenotypic variance. Expected genetic advance was calculated by the method suggested by Johnson et al., (1955) and genetic advance as percentage of mean was calculated by the following formula: 
GA as percentage of mean $=$

$\frac{\text { Genetic advance }}{\text { General mean }} \mathrm{x}$

Correlation coefficients were calculated for all quantitative character combinations at phenotypic, genotypic and environmental level by the formula given by Miller et al., (1958).

$$
r_{X_{i X j}}=\frac{\operatorname{Cov} X_{i} X_{j}}{\sqrt{\left(\operatorname{Var} X_{i}\right)} \cdot \sqrt{\left(\operatorname{Var} X_{j}\right)}}
$$

The significance of phenotypic correlation coefficients was tested against ' $r$ ' values given by Fisher and Yates (1963).

$$
t c a l=\frac{\mathrm{r} \sqrt{n-2}}{\sqrt{1-r^{2}}}
$$

The direct and indirect contribution of various characters to yield were calculated through path coefficient analysis as suggested by Wright (1921) and elaborated by Dewey and Lu (1959).The path coefficients were rated based on the scales given below (Lenka and Mishra, 1973).

$\begin{array}{lll}>1.00 & = & \text { Very high } \\ 0.3-0.99 & = & \text { High } \\ 0.2-0.29 & = & \text { Moderate } \\ 0.1-0.19 & = & \text { Low } \\ 0.0-0.09 & = & \text { Negligible }\end{array}$

\section{Results and Discussion}

\section{Genetic variability parameters}

Genotypic coefficient of variation (GCV) and phenotypic coefficient of variation (PCV)

The magnitude of variation between genotypes was reflected by high values of mean and range for genotypic traits studied
(Table 3). The value of GCV and PCV were obtained high for number of spikelets/panicle, spikelet density, grain yield/plant, fertile 10spikelets/panicle, biological yield /plant and panicle weight/plant. This was in agreement with the finding reported by Shrivastava et al., (2015), Gautam et al., (2016), Devi et al.., (2017), Srujana et al., (2017) and Choudhary et al., (2018). In this study, moderate GCV and PCV were observed for harvest index, thousand grain weight, number of tillers/plant, number of productive tillers/plant, head rice recovery, flag leaf width, stem thickness, spikelet fertility, this was in agreement with Choudhary et al., (2018) and partial agreement with Devi et al., (2017). Similarly, harvest index recorded a high magnitude of GCV and PCV with the findings reported by Shrivastava et al., (2015). Stem length, decorticated grain $1 / \mathrm{b}$ ratio, flag leaf length, plant height, grain breadth, decorticated grain breadth, grain length, decorticated grain length, days to 50\% flowering, panicle length, panicle index, days to maturity, milling percentage and hulling percentage showed low GCV and PCV $(<10 \%)$ which was in agreement with the Hossain et al., (2015) for days to 50\% percent flowering, Sohgaura et al., (2014) for milling percentage, days to $50 \%$ percent flowering and days to maturity.

\section{Heritability and genetic advance analysis}

In our study, most of the characters exhibited high broad sense heritability except flag leaf length which recorded low broad sense heritability. High heritability coupled with high genetic advance showed by thousand grain weight, head rice recovery, spikelet fertility, harvest index, number of spikelets/panicle, fertile spikelets/panicle, spikelet density, grain yield/plant, panicle weight/plant, number of tillers/plant and number of productive tillers/plant. This was partially in consonance with the findings of Dongre et al., (2014); Jha et al., (2014) for 
fertile spikelets/panicle. Similar results were reported by Debnath et al., (2015) for thousand grain weight, panicle weight/plant, fertile spikelets/panicle and grain yield /plant; Devi et al., (2017) for number of productive tillers/plant, fertile spikelets/panicle, thousand grain weight, grain yield /plant and head rice recovery; Vinoth et al., (2016) for fertile spikelets/panicle and fertile spikelets/panicle; Rajesh et al., (2016) for number of productive tillers/plant, fertile spikelets/panicle, number of spikelets/panicle, grain yield /plant and panicle weight/plant; Kumar et al., (2018) for thousand grain weight, spikelet fertility, spikelets per panicle, harvest index, grain yield/plant, biological yield/plant and number of productive tillers/plant.

High heritability coupled with moderate genetic advance were found for grain length, decorticated grain length, days to maturity, days to $50 \%$ flowering, grain width, decorticated grain $1 / b$ ratio, decorticated grain breadth, panicle index, stem length and plant height. This study was in consonance with the findings of Sharma et al., (2014) for days to $50 \%$ flowering and days to maturity.

High heritability accompanied with low genetic advance exhibited by milling percentage and hulling percentage. This was in confirmation with the findings reported by Sohgaura et al., (2014) and Choudhary (2016) for hulling percentage.

\section{Correlation coefficient analysis}

In present investigation, genotypic correlation coefficients were higher in magnitude than phenotypic correlation coefficients in the same direction and magnitude indicated that there is a strong inherent association between each pair of character(s) which might be due to masking or modifying effect of the environment (Table 2).
Grain yield/plant revealed significant and positive association with panicle weight/plant, followed by biological yield/plant, harvest index, number of tillers/plant, number of productive tillers/plant, panicle index, number of fertile spikelets/panicle, number of spikelets/panicle, spikelet fertility and spikelet density. It indicates strong association of these traits with grain yield per plant and selection for such traits will be useful in improving grain yield. A positive correlation between desirable characters is favourable because it helps in simultaneous improvement of both the characters. Similar results were previously confirmed by Ketan and Sarkar (2014), Sohgaura et al., (2014), Dongre et al., (2014), Shrivastava et al., (2015), Yadav et al., (2015), Sameera et al., (2016), Vinoth et al., (2016), Kumar et al., (2017), Onyia et al., (2017), Shamim et al., (2017)and Pratap et al., (2018).

However, negative and highly significant correlation of grain yield/plant was also observed with days to $50 \%$ flowering followed by days to maturity. This finding was in confirmation with the results of Lingaiah et al., (2014), Sohgaura et al., (2014), Onyia et al., (2017) and Hosagoudar et al., (2018).

\section{Path coefficient analysis}

The present investigation revealed that the highest positive direct effect towards grain yield/plant was observed for spikelet density followed by number of tillers/plant, spikelet fertility, panicle weight/plant, decorticated grain length/breadth ratio, flag leaf length, decorticated grain width, milling percentage, panicle length, stem length, stem thickness, days to maturity and thousand grain weight. Similar results were reported by Chakraborty et al., (2010) for Spikelet density and spikelet fertility. Whereas, Kumar et al., (2017) confirmed the highest positive direct effect of spikelet fertility on grain yield/plant. 
Table.1 Parameters of genetic variability for yield, yield attributing and quality traits in NPT lines of rice

\begin{tabular}{|c|c|c|c|c|c|c|c|c|c|}
\hline \multirow{2}{*}{ S.No. } & \multirow{2}{*}{ TRAITS } & \multirow{2}{*}{ Mean } & \multicolumn{2}{|c|}{ Range } & \multicolumn{2}{|c|}{$\begin{array}{l}\text { Coefficient of } \\
\text { variation }\end{array}$} & \multirow{2}{*}{$\begin{array}{c}\mathbf{h}^{2} \\
(\mathbf{b s}) \\
\%\end{array}$} & \multirow{2}{*}{$\begin{array}{c}\text { Genetic } \\
\text { Advance } \\
\text { (GA) }\end{array}$} & \multirow{2}{*}{$\begin{array}{c}\text { Genetic } \\
\text { advance } \\
\text { as } 5 \% \text { of } \\
\text { mean }\end{array}$} \\
\hline & & & Max. & Min. & $\begin{array}{c}\text { GCV } \\
(\%)\end{array}$ & $\begin{array}{l}\text { PCV } \\
(\%)\end{array}$ & & & \\
\hline 1 & DTF & 107.10 & 122.33 & 95.67 & 5.799 & 5.896 & 96.8 & 12.59 & 11.75 \\
\hline 2 & DTM & 134.16 & 154.33 & 124.67 & 5.205 & 5.271 & 97.5 & 14.20 & 10.59 \\
\hline 3 & TPP & 7.35 & 7.84 & 2.50 & 13.166 & 15.525 & 71.9 & 1.69 & 23.00 \\
\hline 4 & PTPP & 7.33 & 7.34 & 2.05 & 13.083 & 15.43 & 71.9 & 1.68 & 22.85 \\
\hline 5 & PH & 101.15 & 121.73 & 83.07 & 7.727 & 9.155 & 71.2 & 13.59 & 13.43 \\
\hline 6 & SL & 75.91 & 93.53 & 58.47 & 9.525 & 11.236 & 71.9 & 12.63 & 16.63 \\
\hline 7 & PL & 25.24 & 29.20 & 22.33 & 5.714 & 7.858 & 52.9 & 2.16 & 8.56 \\
\hline 8 & ST & 5.78 & 7.87 & 4.60 & 11.321 & 13.764 & 67.7 & 1.11 & 19.18 \\
\hline 9 & FLL & 49.74 & 63.00 & 39.00 & 8.648 & 12.446 & 48.3 & 6.16 & 12.38 \\
\hline 10 & FLW & 1.41 & 1.83 & 1.03 & 11.878 & 15.887 & 55.9 & 0.26 & 18.30 \\
\hline 11 & BYPP & 58.02 & 127.67 & 35.00 & 22.07 & 24.134 & 83.6 & 24.12 & 41.58 \\
\hline 12 & PWPP & 26.83 & 56.67 & 16.40 & 21.979 & 24.923 & 77.8 & 10.71 & 39.93 \\
\hline 13 & NSPP & 214.79 & 465.67 & 135.33 & 27.637 & 29.497 & 87.8 & 114.58 & 53.34 \\
\hline 14 & FSPP & 182.60 & 346.67 & 118.33 & 23.014 & 25.718 & 80.1 & 77.47 & 42.43 \\
\hline 15 & SF & 86.33 & 97.43 & 38.30 & 10.385 & 10.843 & 91.7 & 17.69 & 20.49 \\
\hline 16 & SD & 8.51 & 16.03 & 5.67 & 25.437 & 27.924 & 83 & 4.06 & 47.73 \\
\hline 17 & TGW & 24.85 & 34.03 & 14.53 & 16.632 & 16.636 & 99.9 & 8.51 & 34.25 \\
\hline 18 & PI & 74.51 & 84.65 & 62.97 & 5.635 & 6.444 & 76.5 & 7.57 & 10.15 \\
\hline 19 & HI & 34.86 & 60.30 & 24.78 & 17.807 & 18.948 & 88.3 & 12.02 & 34.47 \\
\hline 20 & GL & 9.49 & 11.25 & 7.19 & 6.611 & 6.633 & 99.3 & 1.21 & 13.57 \\
\hline 21 & GW & 2.90 & 3.59 & 2.19 & 7.168 & 7.415 & 93.5 & 0.37 & 14.27 \\
\hline 22 & DGL & 6.59 & 8.14 & 5.98 & 6.145 & 6.196 & 98.3 & 0.81 & 12.55 \\
\hline 23 & DGW & 2.29 & 3.04 & 2.03 & 7.017 & 7.414 & 89.6 & 0.30 & 13.68 \\
\hline 24 & DLBR & 2.91 & 3.44 & 2.08 & 8.876 & 9.303 & 91.0 & 0.52 & 17.45 \\
\hline 25 & HP & 80.07 & 87.72 & 72.01 & 3.131 & 3.566 & 77.1 & 4.45 & 5.66 \\
\hline 26 & MP & 71.64 & 77.70 & 62.72 & 4.833 & 5.099 & 89.8 & 6.91 & 9.44 \\
\hline 27 & HRR & 50.57 & 59.86 & 40.58 & 11.935 & 12.024 & 98.5 & 15.09 & 24.40 \\
\hline 28 & GYPP & 20.02 & 42.20 & 12.47 & 23.26 & 26.129 & 79.2 & 8.54 & 42.66 \\
\hline
\end{tabular}

DTF- Days to 50\% flowering, DTM- Days to maturity, TPP- Number of tillers per plant, PTPP- Productive tillers per plant, PH- Plant height, SL- Stem length, PL- Panicle length, CT- Culm thickness, FLL- Flag leaf length, FLW- Flag leaf width, BYPP- Biological yield per plant, PWPP- Panicle weight per plant, NSPP- Number of spikelet per panicle, FSPP- Fertile spikelet per panicle, SF- Spikelet fertility, SD- Spikelet density, TGWThousand grain weight, PI- Panicle index, HI- Harvest index, GL- Grain length, GW- Grain width, DGLDecorticated grain length, DGW- Decorticated grain width, DLBR- Decorticated length breadth ratio, HP- Hulling percentage, MP- Milling percentage, HRR- Head rice recovery, GYPP- Grain yield per plant. 
Table.2(a) Estimates of phenotypic correlation coefficient for various yield and attributing traits

\begin{tabular}{|c|c|c|c|c|c|c|c|c|c|c|c|c|c|c|}
\hline TRAITS & DTF & DTM & TPP & PTPP & PH & SL & PL & ST & FLL & FLW & BYPP & PWPP & NSPP & FSPP \\
\hline DTF & & $0.940 * *$ & 0.036 & 0.034 & -0.035 & -0.004 & $-0.144 *$ & $0.264 * *$ & $0.228 * *$ & $-0.306 * *$ & -0.134 & $-0.190 * *$ & 0.126 & 0.106 \\
\hline DTM & & & 0.093 & 0.092 & -0.006 & 0.036 & $-0.178 *$ & $0.247 * *$ & $0.182 * *$ & $-0.350 * *$ & -0.083 & $-0.156^{*}$ & 0.068 & 0.064 \\
\hline TPP & & & & $0.999 * *$ & -0.074 & -0.038 & $-0.186 * *$ & -0.009 & -0.114 & -0.122 & $0.381 * *$ & $0.326 * *$ & $-0.220 * *$ & -0.114 \\
\hline PTPP & & & & & -0.077 & -0.041 & $-0.190 * *$ & -0.011 & -0.119 & -0.121 & $0.373 * *$ & $0.315^{* *}$ & $-0.228 * *$ & -0.125 \\
\hline $\mathbf{P H}$ & & & & & & $0.978 * *$ & $0.461 * *$ & 0.118 & $0.371 * *$ & 0.121 & $0.199 * *$ & 0.025 & 0.110 & 0.055 \\
\hline SL & & & & & & & $0.268 * *$ & 0.088 & $0.343^{* *}$ & 0.088 & $0.185^{* *}$ & -0.002 & 0.053 & 0.021 \\
\hline PL & & & & & & & & $0.172 *$ & $0.258 * *$ & $0.189 * *$ & 0.134 & 0.127 & $0.286 * *$ & $0.165^{*}$ \\
\hline ST & & & & & & & & & -0.023 & $-0.201 * *$ & $0.243^{* *}$ & 0.124 & $0.154^{*}$ & 0.060 \\
\hline FLL & & & & & & & & & & $0.324 * *$ & -0.011 & -0.066 & $0.208 * *$ & 0.130 \\
\hline FLW & & & & & & & & & & & -0.082 & 0.009 & $0.203^{* *}$ & $0.240 * *$ \\
\hline BYPP & & & & & & & & & & & & $0.771 * *$ & $0.162 *$ & $0.231 * *$ \\
\hline PWPP & & & & & & & & & & & & & $0.210 * *$ & $0.291 * *$ \\
\hline NSPP & & & & & & & & & & & & & & $0.882 * *$ \\
\hline FSPP & & & & & & & & & & & & & & \\
\hline SF & & & & & & & & & & & & & & \\
\hline SD & & & & & & & & & & & & & & \\
\hline TGW & & & & & & & & & & & & & & \\
\hline PI & & & & & & & & & & & & & & \\
\hline $\mathrm{HI}$ & & & & & & & & & & & & & & \\
\hline GL & & & & & & & & & & & & & & \\
\hline GW & & & & & & & & & & & & & & \\
\hline DGL & & & & & & & & & & & & & & \\
\hline DGW & & & & & & & & & & & & & & \\
\hline DLBR & & & & & & & & & & & & & & \\
\hline HP & & & & & & & & & & & & & & \\
\hline MP & & & & & & & & & & & & & & \\
\hline HRR & & & & & & & & & & & & & & \\
\hline GYPP & $-0.225 * *$ & $-0.194 * *$ & $0.370 * *$ & $0.360 * *$ & -0.004 & -0.022 & 0.077 & 0.078 & -0.073 & 0.061 & $0.733 * *$ & $0.968 * *$ & $0.172 *$ & $0.282 * *$ \\
\hline
\end{tabular}

* Significant at $5 \%$ level; ** Significant at $1 \%$ level 
Table.2(b) Estimates of phenotypic correlation coefficient for various yield and attributing traits (continue)

\begin{tabular}{|c|c|c|c|c|c|c|c|c|c|c|c|c|c|}
\hline TRAITS & SF & SD & TGW & PI & HI & GL & GW & DGL & DGW & DLBR & HP & MP & HRR \\
\hline DTF & $-0.152 *$ & $0.178^{*}$ & $-0.456 * *$ & $-0.191 * *$ & -0.127 & $-0.153^{*}$ & $-0.440 * *$ & $-0.200 * *$ & $-0.263 * *$ & 0.077 & $-0.219 * *$ & $-0.307 * *$ & $0.296 * *$ \\
\hline$\overline{\text { DTM }}$ & -0.095 & 0.128 & $-0.469 * *$ & $-0.180 *$ & $-0.145^{*}$ & -0.136 & $-0.428 * *$ & $-0.149 *$ & $-0.276^{* *}$ & 0.117 & $-0.198 * *$ & $-0.329 * *$ & $0.319 * *$ \\
\hline TPP & $0.276 * *$ & $-0.183 * *$ & 0.080 & $0.242 * *$ & 0.022 & -0.065 & $0.147 *$ & -0.051 & $0.178 *$ & $-0.148^{*}$ & 0.076 & 0.062 & -0.005 \\
\hline PTPP & $0.275 * *$ & $-0.190 * *$ & 0.084 & $0.247 * *$ & 0.019 & -0.062 & $0.147 *$ & -0.049 & $0.179 *$ & $-0.148^{*}$ & 0.075 & 0.062 & -0.008 \\
\hline $\mathrm{PH}$ & -0.099 & -0.016 & $-0.181^{*}$ & $-0.146^{*}$ & $-0.270 * *$ & $-0.165 *$ & -0.113 & 0.006 & -0.091 & 0.062 & -0.129 & -0.100 & $0.172 *$ \\
\hline$\overline{\mathrm{SL}}$ & -0.046 & -0.019 & $-0.203 * *$ & -0.110 & $-0.273 * *$ & $-0.206 * *$ & -0.093 & -0.023 & -0.056 & 0.020 & -0.134 & -0.115 & 0.128 \\
\hline PL & $-0.262 * *$ & 0.009 & 0.027 & $-0.211 * *$ & -0.084 & 0.118 & -0.128 & 0.130 & $-0.182 * *$ & $0.204 * *$ & -0.020 & 0.030 & $0.245^{* *}$ \\
\hline ST & $-0.223 * *$ & 0.112 & $-0.141 *$ & $-0.173^{*}$ & $-0.195 * *$ & 0.135 & $-0.175^{*}$ & 0.135 & -0.117 & $0.192 * *$ & $-0.151 *$ & $-0.175^{*}$ & $0.168 *$ \\
\hline FLL & $-0.220 * *$ & $0.140 *$ & $-0.234 * *$ & -0.078 & -0.104 & $-0.149 *$ & $-0.169 *$ & $-0.180^{*}$ & -0.108 & -0.032 & -0.115 & $-0.158 *$ & $0.156^{*}$ \\
\hline FLW & 0.055 & $0.155^{*}$ & -0.032 & $0.160 *$ & $0.165^{*}$ & -0.126 & -0.033 & $-0.149 *$ & 0.029 & -0.120 & -0.020 & 0.041 & $-0.170 *$ \\
\hline BYPP & 0.097 & 0.132 & 0.033 & 0.001 & $-0.230 * *$ & $0.186^{* *}$ & 0.036 & $0.184 * *$ & -0.026 & 0.136 & 0.077 & 0.012 & 0.119 \\
\hline PWPP & 0.097 & $0.180 *$ & 0.013 & 0.070 & $0.383^{* *}$ & 0.120 & 0.080 & 0.110 & 0.004 & 0.079 & 0.047 & -0.058 & 0.016 \\
\hline NSPP & $-0.486 * *$ & $0.957 * *$ & $-0.497 * *$ & -0.113 & 0.051 & $-0.151^{*}$ & $-0.493 * *$ & $-0.273 * *$ & $-0.456^{* *}$ & $0.174 *$ & 0.059 & -0.054 & $0.176^{*}$ \\
\hline FSPP & -0.033 & $0.881 * *$ & $-0.440 * *$ & 0.009 & 0.114 & -0.130 & $-0.448 * *$ & $-0.271 * *$ & $-0.400 * *$ & 0.130 & 0.091 & 0.037 & $0.200 * *$ \\
\hline SF & & $-0.416^{* *}$ & $0.273 * *$ & $0.316^{* *}$ & 0.109 & 0.052 & $0.228 * *$ & 0.065 & $0.257 * *$ & $-0.162 *$ & 0.095 & $0.236^{* *}$ & -0.034 \\
\hline $\mathrm{SD}$ & & & $-0.536 * *$ & -0.061 & 0.068 & $-0.196 * *$ & $-0.491 * *$ & $-0.319 * *$ & $-0.435 * *$ & 0.131 & 0.063 & -0.075 & 0.113 \\
\hline TGW & & & & 0.086 & -0.020 & $0.540 * *$ & $0.649 * *$ & $0.569 * *$ & $0.511 * *$ & -0.039 & $0.175^{*}$ & $0.309 * *$ & $-0.170 *$ \\
\hline $\mathrm{PI}$ & & & & & $0.455^{* *}$ & $-0.190 * *$ & $0.222 * *$ & $-0.189 * *$ & $0.349 * *$ & $-0.395 * *$ & $0.323 * *$ & $0.378 * *$ & -0.096 \\
\hline $\mathrm{HI}$ & & & & & & $-0.151 *$ & 0.107 & $-0.157^{*}$ & $0.144 *$ & $-0.199 * *$ & 0.116 & 0.072 & -0.136 \\
\hline $\mathrm{GL}$ & & & & & & & $0.170^{*}$ & $0.799 * *$ & -0.133 & $0.587 * *$ & -0.001 & 0.086 & -0.009 \\
\hline $\mathrm{GW}$ & & & & & & & & $0.224 * *$ & $0.649 * *$ & $-0.327 * *$ & $0.153^{*}$ & $0.297 * *$ & -0.103 \\
\hline DGL & & & & & & & & & -0.082 & $0.665 * *$ & -0.015 & 0.034 & -0.118 \\
\hline DGW & & & & & & & & & & $-0.787 * *$ & $0.304 * *$ & $0.277 * *$ & -0.117 \\
\hline DLBR & & & & & & & & & & & $-0.258 * *$ & $-0.212 * *$ & 0.007 \\
\hline $\mathrm{HP}$ & & & & & & & & & & & & $0.755^{* *}$ & $0.186 * *$ \\
\hline MP & & & & & & & & & & & & & $0.332 * *$ \\
\hline HRR & & & & & & & & & & & & & \\
\hline GYPP & $0.167 *$ & $0.155^{*}$ & 0.033 & $0.313 * *$ & $0.480 * *$ & 0.064 & 0.130 & 0.053 & 0.094 & -0.028 & 0.111 & 0.036 & -0.0022 \\
\hline
\end{tabular}

* Significant at $5 \%$ level; ** Significant at $1 \%$ level 
Table.3(a) Estimates of genotypic path coefficient for various yield and quality attributing traits on grain yield/plant

\begin{tabular}{|c|c|c|c|c|c|c|c|c|c|c|c|c|c|c|}
\hline TRAITS & DTF & DTM & TPP & PTPP & PH & SL & PL & ST & FLL & FLW & BYPP & PWPP & NSPP & FSPP \\
\hline DTF & -2.2034 & -2.0794 & -0.0885 & -0.0787 & 0.0736 & -0.0081 & 0.4393 & -0.7273 & -0.7431 & 0.9407 & 0.3116 & 0.4576 & -0.3041 & -0.2714 \\
\hline DTM & 0.7428 & 0.7871 & 0.0833 & 0.0813 & 0.0019 & 0.0408 & -0.1944 & 0.2561 & 0.2006 & -0.3768 & -0.0651 & -0.1313 & 0.0644 & 0.065 \\
\hline TPP & 0.2357 & 0.6209 & 5.8666 & 5.8676 & -0.9299 & -0.5554 & -2.2543 & -0.1044 & -1.5629 & -1.05 & 2.3312 & 1.886 & -1.9633 & -1.3194 \\
\hline PTPP & -0.1851 & -0.535 & -5.1828 & -5.182 & 0.8128 & 0.4776 & 2.0099 & 0.088 & 1.4135 & 0.9432 & -2.0154 & -1.6082 & 1.7687 & 1.2105 \\
\hline $\mathbf{P H}$ & 0.0711 & -0.0051 & 0.3376 & 0.334 & -2.1296 & -2.0969 & -1.0283 & -0.1899 & -1.3335 & -0.5875 & -0.376 & 0.054 & -0.1885 & 0.0209 \\
\hline SL & 0.0046 & 0.0642 & -0.1172 & -0.1141 & 1.2193 & 1.2384 & 0.3993 & 0.0784 & 0.7373 & 0.295 & 0.2082 & -0.0623 & 0.0192 & -0.0711 \\
\hline$\overline{\mathbf{P L}}$ & -0.3955 & -0.49 & -0.7623 & -0.7694 & 0.9578 & 0.6396 & 1.9837 & 0.329 & 0.8103 & 0.5966 & 0.2261 & 0.2272 & 0.7968 & 0.4652 \\
\hline ST & 0.2854 & 0.2812 & -0.0154 & -0.0147 & 0.0771 & 0.0547 & 0.1434 & 0.8645 & -0.0094 & -0.228 & 0.2759 & 0.0996 & 0.1634 & 0.0552 \\
\hline FLL & 0.8534 & 0.645 & -0.6741 & -0.6902 & 1.5844 & 1.5065 & 1.0336 & -0.0275 & 2.5304 & 0.8301 & 0.022 & -0.1471 & 0.8733 & 0.5707 \\
\hline FLW & 0.2406 & 0.2697 & 0.1008 & 0.1026 & -0.1554 & -0.1342 & -0.1695 & 0.1486 & -0.1848 & -0.5634 & 0.0724 & -0.0032 & -0.1385 & -0.1681 \\
\hline BYPP & 0.591 & 0.3457 & -1.6609 & -1.6256 & -0.738 & -0.7027 & -0.4763 & -1.3337 & -0.0363 & 0.5372 & -4.1796 & -3.2268 & -0.386 & -0.5989 \\
\hline$\overline{\text { PWPP }}$ & -1.0122 & -0.8127 & 1.5668 & 1.5125 & -0.1236 & -0.245 & 0.5583 & 0.5614 & -0.2833 & 0.0281 & 3.7627 & 4.8737 & 0.6842 & 1.0075 \\
\hline NSPP & -0.6674 & -0.3958 & 1.6185 & 1.6507 & -0.428 & -0.0751 & -1.9426 & -0.9141 & -1.6691 & -1.1887 & -0.4466 & -0.6789 & -4.8363 & -4.2336 \\
\hline FSPP & -1.1967 & -0.8022 & 2.1852 & 2.2697 & 0.0952 & 0.5575 & -2.2786 & -0.6203 & -2.1912 & -2.8991 & -1.3923 & -2.0086 & -8.5053 & -9.716 \\
\hline$\overline{\text { SF }}$ & -0.882 & -0.55 & 1.8022 & 1.8031 & -0.8644 & -0.5301 & -2.0264 & -1.5267 & -1.9264 & 0.3931 & 0.3284 & 0.309 & -3.1145 & -0.623 \\
\hline SD & 3.1357 & 2.3577 & -4.421 & -4.5214 & -0.2592 & -0.8774 & 2.9936 & 2.4588 & 4.2345 & 2.9433 & 1.1461 & 1.7946 & 15.2393 & 13.9358 \\
\hline TGW & -0.0872 & -0.0894 & 0.0175 & 0.0182 & -0.0402 & -0.0448 & 0.0069 & -0.032 & -0.0633 & -0.0083 & 0.0067 & 0.0028 & -0.1001 & -0.0929 \\
\hline$\overline{\mathbf{P I}}$ & 0.1805 & 0.169 & -0.2839 & -0.2841 & 0.1951 & 0.1488 & 0.3112 & 0.1967 & 0.1082 & -0.1654 & 0.0208 & -0.1004 & 0.1197 & 0.0056 \\
\hline HI & 0.2282 & 0.2611 & -0.016 & -0.0112 & 0.5544 & 0.5518 & 0.2382 & 0.5118 & 0.2292 & -0.3785 & 0.4573 & -0.6044 & -0.0508 & -0.1559 \\
\hline $\mathbf{G L}$ & 0.0281 & 0.0247 & 0.0142 & 0.0136 & 0.0348 & 0.0431 & -0.0275 & -0.0295 & 0.0391 & 0.0309 & -0.0362 & -0.0246 & 0.0282 & 0.0253 \\
\hline$\overline{\text { GW }}$ & 0.4995 & 0.4815 & -0.201 & -0.2012 & 0.134 & 0.1038 & 0.2054 & 0.2059 & 0.247 & 0.0601 & -0.0337 & -0.0903 & 0.593 & 0.5626 \\
\hline DGL & 0.1928 & 0.1453 & 0.0645 & 0.0613 & 0.0025 & 0.0344 & -0.1587 & -0.1614 & 0.2536 & 0.2015 & -0.1922 & -0.1129 & 0.2912 & 0.3084 \\
\hline DGW & -0.7004 & -0.7369 & 0.5934 & 0.5953 & -0.2883 & -0.1746 & -0.6869 & -0.3619 & -0.4103 & 0.1076 & -0.0595 & 0.0664 & -1.2276 & -1.1299 \\
\hline$\overline{\text { DLBR }}$ & 0.2252 & 0.3306 & -0.5241 & -0.5216 & 0.1958 & 0.0567 & 0.7767 & 0.5947 & -0.1242 & -0.4673 & 0.3892 & 0.1805 & 0.4582 & 0.3448 \\
\hline HP & 0.2492 & 0.2213 & -0.1326 & -0.1361 & 0.2198 & 0.2266 & 0.0549 & 0.1778 & 0.2411 & -0.0272 & -0.0964 & -0.1115 & -0.074 & -0.112 \\
\hline MP & -0.7041 & -0.7424 & 0.2166 & 0.2176 & -0.2912 & -0.3318 & 0.0853 & -0.4093 & -0.5982 & 0.118 & 0.0437 & -0.0745 & -0.1289 & 0.0905 \\
\hline HRR & 0.0221 & 0.0238 & 0.0004 & 0.0001 & 0.0145 & 0.0107 & 0.025 & 0.0162 & 0.0161 & -0.0169 & 0.0098 & 0.0025 & 0.0138 & 0.0163 \\
\hline GYPP & -0.2482 & -0.2102 & 0.3877 & 0.3772 & -0.0747 & -0.085 & 0.0213 & 0.0498 & -0.0752 & 0.0682 & 0.7189 & 0.9689 & 0.0955 & 0.1921 \\
\hline
\end{tabular}


Table.3(b) Estimates of genotypic path coefficient for various yield and quality attributing traits on grain yield/plant (continue)

\begin{tabular}{|c|c|c|c|c|c|c|c|c|c|c|c|c|c|}
\hline TRAITS & SF & SD & TGW & PI & HI & GL & GW & DGL & DGW & DLBR & HP & MP & HRR \\
\hline DTF & 0.3527 & -0.4411 & 1.0203 & 0.4983 & 0.2967 & 0.3528 & 1.0275 & 0.446 & 0.6348 & -0.1946 & 0.5464 & 0.739 & -0.6635 \\
\hline$\overline{\text { DTM }}$ & -0.0786 & 0.1185 & -0.374 & -0.1667 & -0.1213 & -0.1108 & -0.3538 & -0.1201 & -0.2386 & 0.102 & -0.1733 & -0.2783 & 0.2557 \\
\hline TPP & 1.9186 & -1.6558 & 0.5447 & 2.0863 & 0.0555 & -0.4751 & 1.1009 & -0.3974 & 1.432 & -1.2057 & 0.7739 & 0.6052 & 0.0282 \\
\hline PTPP & -1.6955 & 1.4958 & -0.5015 & -1.8443 & -0.0342 & 0.4004 & -0.9735 & 0.3338 & -1.269 & 1.0601 & -0.7016 & -0.5371 & -0.0084 \\
\hline PH & 0.334 & 0.0352 & 0.4548 & 0.5205 & 0.6968 & 0.4226 & 0.2664 & 0.0056 & 0.2526 & -0.1636 & 0.4657 & 0.2954 & -0.4223 \\
\hline SL & -0.1191 & -0.0694 & -0.295 & -0.2308 & -0.4033 & -0.3043 & -0.1201 & -0.0447 & -0.089 & 0.0276 & -0.2792 & -0.1957 & 0.1811 \\
\hline $\mathbf{P L}$ & -0.7295 & 0.3791 & 0.0731 & -0.7735 & -0.2788 & 0.3102 & -0.3803 & 0.3306 & -0.5605 & 0.6043 & -0.1084 & 0.0806 & 0.6777 \\
\hline ST & -0.2395 & 0.1357 & -0.1469 & -0.213 & -0.2611 & 0.1454 & -0.1662 & 0.1465 & -0.1287 & 0.2016 & -0.153 & -0.1685 & 0.1913 \\
\hline FLL & -0.8845 & 0.6841 & -0.8505 & -0.3429 & -0.3422 & -0.5629 & -0.5834 & -0.6738 & -0.4271 & -0.1233 & -0.607 & -0.721 & 0.5572 \\
\hline FLW & -0.0402 & -0.1059 & 0.0249 & -0.1168 & -0.1258 & 0.099 & 0.0316 & 0.1192 & -0.0249 & 0.1033 & -0.0153 & -0.0317 & 0.13 \\
\hline BYPP & -0.2491 & -0.3058 & -0.1493 & 0.1089 & 1.128 & -0.8622 & -0.1316 & -0.8433 & 0.1023 & -0.638 & -0.4008 & -0.0871 & -0.5588 \\
\hline PWPP & 0.2733 & 0.5584 & 0.0732 & 0.6132 & 1.7383 & 0.682 & 0.4107 & 0.5777 & 0.1331 & 0.3449 & 0.5407 & -0.1731 & 0.1665 \\
\hline NSPP & 2.7333 & -4.7053 & 2.5718 & 0.7255 & -0.145 & 0.7772 & 2.6772 & 1.4784 & 2.4422 & -0.869 & -0.3562 & 0.2969 & -0.9085 \\
\hline FSPP & 1.0985 & -8.6444 & 4.7928 & 0.0678 & -0.894 & 1.4026 & 5.1031 & 3.1462 & 4.5157 & -1.314 & -1.0825 & -0.4187 & -2.1586 \\
\hline SF & 5.5107 & -2.7971 & 1.5706 & 2.0433 & 0.5694 & 0.3036 & 1.3978 & 0.3522 & 1.5276 & -0.948 & 0.5718 & 1.4094 & -0.1962 \\
\hline SD & -7.9504 & 15.6633 & -9.238 & -1.2394 & 0.8007 & -3.3444 & -8.867 & -5.6832 & -7.7499 & 2.1298 & 1.2766 & -1.3787 & 1.9325 \\
\hline TGW & 0.0537 & -0.111 & 0.1883 & 0.0179 & -0.0039 & 0.102 & 0.1267 & 0.108 & 0.1016 & -0.0077 & 0.0376 & 0.0616 & -0.0323 \\
\hline $\mathbf{P I}$ & -0.296 & 0.0632 & -0.0757 & -0.7982 & -0.4287 & 0.1745 & -0.2112 & 0.1741 & -0.3156 & 0.3541 & -0.339 & -0.365 & 0.0905 \\
\hline HI & -0.1751 & -0.0866 & 0.0347 & -0.9102 & -1.6946 & 0.2738 & -0.1958 & 0.3036 & -0.2892 & 0.3971 & -0.3292 & -0.1834 & 0.227 \\
\hline GL & -0.0097 & 0.0375 & -0.0952 & 0.0384 & 0.0284 & -0.1756 & -0.0304 & -0.1419 & 0.0252 & -0.1086 & 0.0014 & -0.0153 & 0.0017 \\
\hline GW & -0.2717 & 0.6064 & -0.7209 & -0.2834 & -0.1238 & -0.1857 & -1.0712 & -0.2492 & -0.7587 & 0.3812 & -0.1845 & -0.3428 & 0.1169 \\
\hline DGL & -0.0609 & 0.3456 & -0.5465 & 0.2078 & 0.1706 & -0.7697 & -0.2216 & -0.9525 & 0.0812 & -0.6572 & 0.0228 & -0.0308 & 0.1162 \\
\hline DGW & 0.6739 & -1.2028 & 1.3126 & 0.9612 & 0.4148 & -0.3484 & 1.7219 & -0.2072 & 2.431 & -1.8873 & 0.843 & 0.7376 & -0.3171 \\
\hline DLBR & -0.4386 & 0.3467 & -0.1044 & -1.1312 & -0.5975 & 1.5769 & -0.9075 & 1.7595 & -1.9795 & 2.5498 & -0.7546 & -0.5911 & 0.0288 \\
\hline HP & -0.1043 & -0.0819 & -0.2009 & -0.4269 & -0.1953 & 0.0081 & -0.1731 & 0.024 & -0.3485 & 0.2975 & -1.0051 & -0.7597 & -0.1641 \\
\hline $\mathbf{M P}$ & 0.537 & -0.1848 & 0.6866 & 0.96 & 0.2273 & 0.1832 & 0.6719 & 0.0678 & 0.637 & -0.4867 & 1.5868 & 2.0995 & 0.6651 \\
\hline HRR & -0.0026 & 0.009 & -0.0126 & -0.0083 & -0.0098 & -0.0007 & -0.008 & -0.0089 & -0.0096 & 0.0008 & 0.012 & 0.0232 & 0.0733 \\
\hline GYPP & 0.1404 & 0.0865 & 0.0369 & 0.3634 & 0.4673 & 0.0747 & 0.1408 & 0.0512 & 0.1277 & -0.0496 & 0.189 & 0.0704 & 0.0099 \\
\hline \multicolumn{14}{|c|}{ R SQUARE $=0.9835$} \\
\hline
\end{tabular}


Number of tillers/plant had positive direct effect on grain yield/plant this result collaborated with the finding of Dongre et al., (2014). While, panicle weight/plant exhibited positive direct effect on grain yield/plant was in agreement with the result of Kumar et al., (2014) and Yadav et al., (2015). Decorticated grain length/breadth ratio and milling percentage recorded positive direct effect on grain yield/plant was in confirmation with the findings of Haque et al., (2014) and Devi et al., (2017) respectively.

Panicle length had positive direct effect on grain yield/plant was in agreement with the results of Lingaiah et al., (2014) and Pratap et al., (2018) with positive direct effect. Whereas, stem length recorded positive direct on grain yield/plant was in confirmation with the findings of Dongre et al., (2014) and Kumar et al., (2014).Days to maturity had positive direct effect on grain yield/plant. This was in consonance with the finding of Sohgaura et al., (2014), Karim et al., (2016), Kumar et al., (2017) and Shamim et al., (2017). The positive direct effect of thousand grain weight was in confirmatory with the results of Dongre et al., (2014), Lingaiah et al., (2014), Sohgaura et al., (2014), Debnath et al., (2015), Devi et al., (2017) and Pratap et al., (2018).

In conclusion, the mean sum of squares due to genotypes for all the characters was highly significant for all the traits under study and indicated that the genotypes possessed inherent genetic differences. The values of PCV for all the traits were found to be more than GCV and very small difference was present in between GCV and PCV revealing little influence of environment for their expression. High heritability accompanied with high genetic advance was observed for the traits thousand grain weight $(\mathrm{g})$, head rice recovery (\%), spikelet fertility (\%), harvest index $(\%)$, number of spikelets/panicle, fertile spikelets/panicle, spikelet density, grain yield /plant (g), panicle weight/plant (g), number of tillers/plant and number of productive tillers/plant. It indicates that the heritability is most likely due to additive gene effect and direct selection for these traits may be effective. Based on the results from correlation and path coefficient analysis, it is concluded that number of tillers/plant, spikelet fertility and panicle weight/plant showed positive correlation with grain yield and at same time exhibited high positive direct effect towards yield. Therefore, they seem to be primary yield contributing character and thus can be used as direct selection to improve genetic yield potential of rice.

\section{References}

Allard, R.W. 1960. Principles of Plant Breeding. 1st Edn., John Wiley and Sons. Incorporation New York. $\mathrm{p} 485$.

Burton, G.W. 1952. Quantitative inheritance in grasses. Proc. 6th Int. Grassland Cong. 1:227-283.

Chakraborty, S., Das, P.K., Guha, B., Sarmah K.K., and Barman, B. 2010. Quantitative genetic analysis for yield and yield components in boro rice (Oryza sativa L.). Not. Sci. Biol., 2(1): 117-120.

Choudhary, P., Mishra, D.K., Koutu, G.K., Pachori, A., and Singh S.K. 2018.Interrelationship of Yield and Quality Attributing Traits in JNPT Lines of Rice. International Journal of Bio-Resource \& Stress Management, 9(3): 330-340.

Choudhary, P., Patle, N., and Mishra, D.K. 2016. Study of Incorporated Diversity in Phenotypic Features of JNPT Lines of Rice. Quarterly Research Journal of Plant \& Animal Sciences/Bhartiya Krishi Anusandhan Patrika 31(3): 171176. 
Cochran, G.W., and Cox, G.M. 1950. Experimental designs. John Wiley and Sons, New York 45-67.

Debnath, K., Das, B., Sikder, S., and Sarkar, K.K. 2015.Assessment of genetic variability character association and path coefficient for yield and its component characters in rice.The Ecoscan. 9(1\&2): 455-459.

Devi, K.R., Chandra, B.S., Lingaiah, N., Hari, Y., and Venkanna, V. 2017. Analysis of variability, correlation and path coefficient studies for yield and quality traits in rice (Oryza sativa L.). Agri. Sci. Digest 37(1): 1-9.

Dewey, D.R., and Lu, K.H. 1959. A correlation and path coefficient analysis of components of crested wheat grass seed production. Agron. Journal 51: 515 $-518$.

Dongre, P.R., Mishra, D.K., Koutu, G.K., and Singh, S.K. 2014. Estimation of genetic variability and correlation for grain yield and its components in RILs derived population of rice. JNKW Research Journal 48(1): 55-59.

FAO. 2018. Rice Market Monitor, April 2018, Volume XXI - Issue No. 1

Gautam, A., Suresh, J., and Madhav, M.S. 2016. Study of Genetic Variability in Genotypes of Rice (Oryza sativa L.). Research Journal of Agricultural Sciences, 7(1): 235-236.

Haque, S., Pradhan, S.K., Anandan, A., Dash, S.K., Swain, P., and Singh, O.N. 2014.Effectiveness of traits for direct selection under severe drought stress condition in rice. The International Journal of Science \&Technology, Page 2(9): 92-96.

Hosagoudar, G.N., and Kovi, B.S. 2018. Evaluation of Advanced Varietal Rice Genotypes for Leaf Blast Reaction, Genotypic Performance and Correlation Studies. Int. Journal Curr.Microbiol. App. Sci. 7(4): 75-81.
Hossain, S., Haque, M.D., and Rahman, J. 2015.Genetic variability, Correlation and Path Coefficient Analysis of Morphological Traits in some Extinct Local Aman Rice (Oryza sativa L.) Rice Research 4(1): 100-158.

Jha, V.B., Sharma, A.K., and Birendra, S. 2014. Genetic variability for different quantitative traits in early rice. Annals of Agri Bio Research 19(1): 25-28.

Johnson, H.W., Robinson, H.F., and Comstock, R.E. 1955. Estimation of genetic and environmental variability in soybean. Agronomy Journal, 47:314318.

Karim, M.J., Jesmin, F., Ali, M.H., Islam, M.N., and Mahmud, E. 2016.Correlation and Path Coefficient Analysis for Quantitative Characters in T. Aman Rice. Ecofriendly Agril. Journal 9 (11): 79-82.

Ketan, R., and Sarkar, G. 2014. Studies on variability, heritability, genetic advance and path analysis in some indigenous Aman rice (Oryza sativa L.). Journal of Crop and Weed 10(2): 308-315.

Khan, N., and Naqvi, F.N. 2011. Heritability of morphological traits in bread wheat Advanced lines under irrigated and nonirrigated conditions. Asian Journal of Agriculture Science 3(3): 215-222.

Kumar, S., Bhuvaneswari, S., Devi, E.L., Sharma, S.K., Ansari, M.A., Singh, I.M., Singh, Y.R., and Prakash, N. 2017. Estimation of genetic variability, correlation and path analysis in short duration rice genotypes of Manipur. Journal of AgriSearch 4(2): 112-118.

Kumar, S., Chauhan, M.P., Tomar, A., and Kasana, R.K. 2018. Coefficient of variation (GCV \& PCV), heritability and genetic advance analysis for yield contributing characters in rice (Oryza sativa L.). Journal of Pharmacognosy and Phytochemistry 7(3): 2161-2164.

Kumar, V., Koshta, N., Sohgaura, N., and 
Koutu, G.K. 2014.Genetic evaluation of RILs population for yield and quality attributing traits in Rice (Oryza sativa L.) Journal of Agricultural technology 1(1): 43-51.

Lenka, D., and Mishra, B. 1973.Path coefficient analysis of yield in rice varieties. Indian Journal Agric. Sci. 43: $376-379$.

Lingaiah, N., Venkanna, V., Cheralu, C., and Satish Chandra, B. 2014. Correlation and path analysis for yield and yield attributes in mid early group genotypes of rice (Oryza sativa L.). International Journal of Innovative Science, Engineering and Technology 1(9): 7982.

Miller, D.A., Williams, J.C.I., Robinson, H.F., and Comstock, K.B. 1958. Estimate of genotypic and environmental variances and covariance in upland cotton and their implication in selection. Agron. Journal 50: 126-131.

Onyia, V.N., Okechukwu, E.C., Atugwu, A.I., and Akpan, N.M. 2017. Genetic Variability Studies on Twelve Genotypes of Rice (Oryza sativa L.) for Growth and Yield Performance in South Eastern Nigeria. Notulae Scientia Biologicae 9(1): 110-115.

Pratap, A., Bisen, P., Loitongbam, B., and Singh, P.K. 2018.Assessment of Genetic Variability for Yield and Yield Components in Rice (Oryza sativa L.) Germplasms. International Journal of Bio-resource and Stress Management, 9(1): 87-92.

Rajesh, T., Radhakrishnan, V.V., Kumari, K.T., Sreenivasan, E., Ibrahim, K.K., and Latha, A. 2016. Variability and Genetic Parameter Analysis in Kerela Rice (Oryza sativa L.) varieties. Advances in Life Sciences 5(6): 23522355.

Sameera, S., Srinivas, T., Rajesh, A.P., Jayalakshmi, V., and Nirmala, P.J.
2016. Variability and path co-efficient for yield and yield components in rice. Bangladesh Journal of Agricultural Research. 41(2): 259-271.

Shamim, M.Z., Sharma, V.K., Manzar, H., and Bhushan, S. 2017. Grain Yield Components Analysis in Locally Adapted Rice Varieties. International Journal of Agriculture, Environment and Biotechnology. 10(4): 435-442.

Sharma, S., Singh, S., Kuldeep, R., and Beniwal, D.C. 2014. Genetic variability and heritability estimates of rice new plant types lines for various quantitative traits. Agriculture for Sustainable Development. 2(2): 137-140.

Shrivastava, A., Koutu, G.K., Mishra, D.K., and Singh, S.K. 2015. Characterization of JNPT lines of rice (Oryza sativa L.). Plant Archives. 15(1): 397-403.

Sivasubramanian, J., and Madhavamenon, P. 1973. Genotypic and phenotypic variability in rice. Madras Agric. Journal 12: 15-16.

Sohgaura, N., Mishra, D.K., Koutu, G.K., and Kumar, V. 2014.Genetic Evaluation of Inter SubSpecific Derived RILs population for Yield and Quality Attributes in Rice. Trends in Bio sciences 7(18): 2631-2638.

Srujana, G., Suresh, B.G., Lavanya, G.R., Ram, B.J., and Sumanth, V. 2017. Studies on Genetic Variability, Heritability and Genetic advance for yield and quality components in rice (Oryza sativa L.). Journal of Pharmacognosy and Phytochemistry, 6(4): 564-566.

Vinoth, R., Shivramakrishnan, R., Sivaji, M., Tamilkumar, P., Binod, K., and Shailesh, M. 2016. Genetic analysis and correlation studies for grain yield in rice (Oryza sativa L.) under the Allahabad agro climatic region. International Journal of Forestry and Crop Improvement. 7(1): 93-100. 
Wright, S. 1921. Correlation and Causation. Journal Agric. Sci. 20: 55 -587.

Yadav, R.B., Mishra, D.K., Koutu, G.K., Singh, S.K., and Shrivastava, A. 2015. Genetic analysis of Indica-Japonica derived rice NPT lines for yield and yield attributing traits under rainfed situation. JNKVV Res. Journal 49(1): 26-31.

\section{How to cite this article:}

Shivangi Rahangdale, Yogendra Singh, G.K. Koutu and Tiwari, S. 2019. Genetic Variability, Correlation and Path Coefficient Studied for Yield and Quality Traits in JNPT Lines of Rice (Oryza sativa L.). Int.J.Curr.Microbiol.App.Sci. 8(10): 1025-1037.

doi: https://doi.org/10.20546/ijcmas.2019.810.120 